\title{
Brain metastases: increasingly precision medicine-a narrative review
}

\author{
Michael Jerome McKay ${ }^{1,2}$ \\ ${ }^{1}$ Northern Cancer Service, North West Cancer Centre, Burnie, Tasmania, Australia; ${ }^{2}$ The University of Tasmania, Rural Clinical School, Northwest \\ Regional Hospital, Burnie, Tasmania, Australia \\ Correspondence to: Professor Michael Jerome McKay, MD, PhD. Northern Cancer Service, North West Cancer Centre, Burnie, Tasmania 7320, \\ Australia. Email: michael.mckay@ths.tas.gov.au.
}

\begin{abstract}
Objective: To broadly review the modern management of brain metastases.
Background: Brain metastases are the commonest neurological manifestation of cancer and a major cause of morbidity in cancer patients. Brain metastases are increasing in frequency, as a result of longer life expectancy of cancer patients, more sensitive methods for brain metastasis detection and an ageing population. The proportional incidence of brain metastases according to cancer of origin, from greatest to least, is lung cancer, melanoma, renal, breast and colorectal cancers. Patients with lung cancer and melanoma are most likely to have brain metastases at diagnosis. Brain metastases cause a variety of symptoms, depending on their size and location, whether they cause mass effect and oedema, compression of the brain parenchyma, or focal neurological deficits. The major differential diagnoses of brain metastases include primary tumours and vascular/inflammatory lesions. Prognosis is dependent on the site, number and volume of lesions, the patients' performance status, age and the activity and extent of extracranial disease.
\end{abstract}

Methods: English literature articles in PubMed from 1950 to June 2021 were reviewed. Article bibliographies provided further references.

Conclusions: Treatment of brain metastasis patients has moved from considering them as a homogenous population of patients, to individualised treatment. In those brain metastases patients of satisfactory performance status with a solitary lesion, especially one in a non-eloquent/accessible area causing significant mass effect and/or raised intracranial pressure or for whom the diagnosis is in doubt (histology needed), surgical resection is usually the treatment of choice. For multiple brain metastases, radiotherapy with or without systemic therapies are usually employed. For relatively fit patients with limited numbers of brain metastases (e.g., 4 or less), stereotactic radiosurgery is standard of care. Current clinical trials are testing the efficacy of stereotactic treatment alone for $>4$ brain metastases (although it is increasingly used for such patients in many centres) as well as integration of local therapies with targeted and immunological therapies in appropriately selected cases. In certain circumstances, cranial irradiation can be omitted.

Keywords: Brain; metastases; surgery; radiotherapy; targeted therapies

Submitted Jul 14, 2021. Accepted for publication Oct 12, 2021.

doi: 10.21037/atm-21-3665

View this article at: https://dx.doi.org/10.21037/atm-21-3665

\section{Introduction}

Brain metastases (BM) are the commonest neurological manifestation of cancer and a major cause of morbidity in cancer patients. It was estimated that in 2020, there were just under 150,000 cases of cancer in Australia, and just under 50,000 cancer-attributable deaths (1). Of the 150,000 cancer cases, over $20 \%$ will develop BM (>30,000 patients) (2). $\mathrm{BM}$ are increasing in frequency, as a result of longer life expectancy of cancer patients, more sensitive methods for $\mathrm{BM}$ detection and an ageing population.

The proportional incidence of BM varies according to cancer of origin; from greatest to least: lung cancer, 
melanoma, renal, breast and colorectal cancers (3). Patients with lung cancer [both small cell lung cancer (SCLC), and non-small cell lung cancer (NSCLC)] and melanoma are most likely to have BM at diagnosis (4). Furthermore, the molecular subtypes of different cancers effects BM incidence (and treatment-see below). Patients with melanomas with $\mathrm{BRAF}^{\mathrm{V} 00 \mathrm{E}}$ or NRAS mutations are more likely to develop BM $(5,6)$, as are patients with EGFRmutant NSCLC variants (7) and patients with HER2+ and triple-negative molecular subtypes of breast cancer (5).

$\mathrm{BM}$ can manifest pleiotropic clinical features. These depend on their size and location, whether they cause mass effect and oedema, compression of the brain parenchyma or focal neurological deficits. The major differential diagnoses of BM include primary tumours and vascular/inflammatory lesions. Intracranially, the most common sites for $\mathrm{BM}$ are the cerebral hemispheres (80\%), cerebellum (15\%) and basal nuclei/brainstem (5-10\%) (8). Most hemispheric lesions occur at the grey-white matter interface. Prognosis is dependent on the site and number of lesions, the patients' performance status, age and the activity and extent of extracranial disease. These factors have been incorporated by the Radiation Therapy Oncology Group (RTOG) into a useful prognostic index, the Graded Prognostic Assessment (GPA) scoring system (9). GPA scores closely correlate with overall survival. Since the extent of extracranial disease is commonly a major prognosticator, this can explain why even successful treatment of intracranial disease may not impact overall outcome: over $50 \%$ of patients with BM die of uncontrolled extracranial disease (10). Here, the major focus of BM management is the maintenance of quality and perhaps quantity of life and hopefully, avoidance of a protracted neurological death.

I present the following article in accordance with the Narrative Review reporting checklist (available at https:// dx.doi.org/10.21037/atm-21-3665).

\section{Objective}

Treatment of patients with BM has moved from considering them as a homogenous population of patients, to individualising treatment. Despite advances in surgery, radiotherapy and systemic therapies, the prognosis of patients with BM remains poor, with median survivals, depending on prognostic factors, of a few months to around a year $(4,11)$. However, a notable exception is the more protracted survival in subsets of patients selected for appropriate molecular therapies, where survival can be as high as multiple years $(5,12)$. In patients of poor performance status, best supportive care is often appropriate. In those BM patients of satisfactory performance status with a solitary lesion, especially one in a non-eloquent, accessible area causing significant mass effect and/or raised intracranial pressure or for whom the diagnosis is in doubt (histology needed), surgical resection is usually the treatment of choice. This has historically been followed by radiotherapy (RT) to the whole brain (WBRT), which can significantly increase local control (13), but has a lesser impact on overall survival.

More recently, in an attempt to reduce the neurocognitive and QoL deficits attributed to WBRT (see below), for limited numbers of BM (e.g., 4 or less), stereotactic radiotherapy (SRT) [or stereotactic radiosurgery (SRS), which is a single fraction of SRT] has been applied. (The term SRS will subsequently be used throughout this article to cover both SRS and SRT). Current clinical trials are testing the efficacy of SRS alone for $>4$ BM, as well as postoperative SRS ('cavity SRS'). Ongoing SRS studies are also examining integration of SRS with targeted systemic therapies that cross, and are retained within, the bloodbrain-barrier and blood-tumour barrier and/or which generate an immune response.

\section{Methods}

English literature articles in PubMed from 1950 to June 2021 were reviewed. Article bibliographies provided further references. Keywords were: brain; metastases; metastasis; surgery; radiotherapy; targeted therapies; immunotherapy.

\section{Symptomatic treatment}

The main symptomatic treatments in BM patients are anticonvulsants and steroids. Seizures occur in up to $25 \%$ of BM patients (14). A meta-analysis concluded that prophylactic use of anti-seizure medications in BM patients without seizures did not reduce their incidence and they are hence not routinely recommended (15).

Steroids, predominantly dexamethasone, are commonly used in BM patients to cause decompression from cerebral oedema in the short-term. They often lead to dramatic symptomatic and physical responses. However, beneficial effects of steroids are impermanent and patients should, clinical circumstances allowing, be weaned off them as early as practicable. Their role in avoiding RT-associated 
oedema is variable: many patients do not develop oedema during WBRT and steroids are not required. For SRS, short courses of steroids are often used. Typical dosing is around $16 \mathrm{mg} /$ day in divided doses, weaning as appropriate, usually within 2 weeks of initiation (16). This should avoid significant steroid-related morbidities such as poor glycaemic control, weight gain, proximal myopathy, impaired wound healing and mood changes. Another consideration which encourages judicious steroid use is treatment with systemic immune checkpoint inhibitor therapies whose effects can be diluted or abrogated by steroids (17).

\section{Local therapies: surgery}

In patients of appropriate fitness (e.g., Karnofsky Performance Score $\geq 70$ ) (18), surgery is usually employed for those with a single large BM. Surgery can establish a histopathological diagnosis, provide tissue for molecular analyses, achieve decompression and reduce cerebral mass effect. The morbidity and mortality of surgical resection is falling and improved outcomes are associated with higherthroughput surgical centres (19). More recently, surgical resection of BM has been followed by focussed RT to the excision cavity—see below.

However, surgery alone, even in contemporary series, has been found insufficient for achieving high rates of BM local control. When combined with adjuvant RT, surgery prolongs survival, as shown in two randomized trials of solitary BM $(20,21)$. Some data indicate that up to three BMs can be successfully treated with surgical resection (22). As part of the modern management of BM, tissue obtained from resection can be used for molecular analyses, to guide subsequent selection of targeted therapies, depending on primary tumour type; such BM analyses are important, since major molecular differences may exist between the primary tumour, extracranial metastases and BM, for which the term 'molecular divergence' has been used (5). Minimally invasive surgery is increasingly used to obtain tissue for analysis.

\section{Local therapies: WBRT}

In the United States alone, around 200,000 patients receive WBRT each year for BM (23). WBRT has been employed for decades as the cornerstone of treatment of BM. It is a simple, cost-effective and straightforward treatment, provides good palliation and local lesion control as well as good distant in-brain control. Non-randomised data indicates that WBRT modestly extends survival from historical levels of 1-2 to 3-6 months (8). In at least two randomised Phase III trials, WBRT was successful in abrogating neurological deaths and was associated with much lower rates of intracranial recurrence compared to focal therapies (surgery, SRS) alone $(13,24)$.

However, not all clinical trials have demonstrated clearcut clinical benefits of WBRT. The QUARTZ (Quality of Life after Treatment for Brain Metastases) phase III study randomised 538 poor-prognosis NSCLC BM patients, unfit for surgery or SRS, to best supportive care or WBRT (25). The primary endpoint was quality-adjusted life years. There was no difference in quality-adjusted life years, survival or steroid use in either arm. This multicentre trial was criticised because of the extremely poor prognosis in both arms, where few modern BM cases would have met the eligibility criteria. However, from these data it could be concluded that in poor-prognosis NSCLC patients with BM, WBRT conferred no benefit compared with best supportive care. Likewise, an Australasian phase 3 study of 1-3 BM in metastatic melanoma patients showed no increase in in-brain control rates or survival by the addition of WBRT (26).

Further, omission of WBRT after focal therapies such as surgery or SRS did not impair overall survival in a number of randomised trials $(24,27,28)$. WBRT is also associated with impairment of both neurocognitive function (NCF), as well as leading to deficits in quality of life (QoL) (27-29). These adverse effects of WBRT have become more evident using the sensitive NCF/QoL measurement instruments of the modern era.

A number of strategies have been used to improve the toxicity profile of WBRT. The hippocampus subserves higher functions in terms of acute memory and cognition. In preclinical investigations, radiation doses as low as 2 Gy have been shown to cause hippocampal neural progenitor cell dysfunction (30,31). To attempt to reduce some of the neurocognitive decline associated with WBRT, hippocampal avoidance was developed. Using advanced RT with volumetric arc therapy (VMAT), the region surrounding the hippocampi can be selectively dose-spared during WBRT, using hippocampal avoidance (HA-WBRT) [(32); MJM, In the press)]. The HA-WBRT technique appears not to be associated with macroscopic recurrences within the proximity of the hippocampi, in MRI-screened cases (33). The RTOG 0933 Phase II single arm study of WBRT in patients with BM from epithelial malignancies (33) showed that cognition decline was reduced using HA-WBRT when 
compared with historical WBRT controls. Specifically, HA-WBRT cases had less reduction in the Hopkins Verbal Learning Test - Revised (HVLT-R) delayed recall test scores than controls ( $7 \%$ at 4 months of $30 \%$ at 4 months in WBRT controls, $\mathrm{P}<0.001)$. Concerns have however been raised that the historical controls were different from the study subjects, for example, their median survivals were relatively lower than the study participants. The entire HA-WBT approach has also been questioned, in that the hippocampus does not subserve all memory and cognition functions and so its sparing may not significantly impact cognitive decline in general (34).

Some investigators have examined HA-WBRT in the context of prophylactic cranial irradiation (PCI), a standard component of therapy in limited disease small cell lung cancer (SCLC) patients. Redmond et al. [2017] (35) found that of 20 SCLC patients treated with HA-WBRT PCI, only $20 \%$ developed asymptomatic brain metastases at 9 months, and in none was there a decline in performance in any of a number of neurocognitive tests. They also noted two cases where recurrence occurred in the underdosed (avoidance) region. Further randomised Phase III trials on the topic of HA-WBRT are underway. Other investigators have pointed out that damage to the genu of the corpus callosum, both in cancer and other patients, is related to impairment of a broad range of NCFs, similar to those also observed in WBRT-treated patients. Analogous to HAWBRT, a clinical trial of genu-avoidance using VMAT WBRT is underway (NCT03223922).

A high ratio of NMDA to GABA receptors is important for neuronal health. Memantine is a NMDA receptor antagonist and thereby neuroprotective agent. It has been postulated that RT may adversely affect this balance: in the NRG-CC001 phase III trial of memantine plus either WBRT or HA-WBRT for BM patients (36) the primary endpoint was preservation of memory as assessed by HVLT-R testing. With a total of 518 patients randomly assigned, the HA-WBRT arm had significantly lower cognitive decline than the WBRT arm. This was seen predominantly as a result of reduced deterioration of executive functioning at 4 months and memory and learning at 6 months. Patient-reported symptoms were also better in the HA-WBRT arm, but there were no differences in overall survival or intracranial progression-free survival in either arm. For patients with BM, but without metastases in the hippocampal region, these investigators suggested that HA-WBRT + memantine is a new standard of care.

Donepezil is an acetylcholinesterase inhibitor used in the treatment of Alzheimer's Disease. In a phase III investigation, it showed some promise (modest improvements in memory) in patients with pre-RTdetermined cognitive impairment (37). A single-arm phase 2 study evaluated Ginko biloba in patients receiving WBRT or partial cranial irradiation and found improved NCF with increasing time from treatment with this agent (38).

\section{Local therapies: stereotactic radiation}

There has been a movement away from WBRT for treatment of a small number of BMs (e.g., <4), because of its toxicity, as addressed above. SRS is a form of focussed RT, where multiple often non-coplanar beams criss-cross the BM target, with extremely sharp dose gradients. SRS is delivered in one or a few fractions; fractionated regimens are often employed for BM lesions adjacent to or within particularly sensitive regions of the brain (e.g., brainstem). SRS alone however, has a relatively high distant in-brain failure rate, occurring without a decrease in overall survival, partly because of effective salvage in patients undergoing close MRI surveillance. Hence, WBRT was historically often given after SRS, as some, but not all (26) trials showed a decrease in distant in-brain failure rate when WBRT was added. Randomised trials comparing the safety and efficacy of SRS alone versus SRS plus WBRT showed, in the WBRT arms, a decline in NCF (e.g., HVLT-R scores) and QoL (26-28,39). The Brown et al. [2016] study (27) showed neurocognitive decline in any one of a number of tests, from > one SD from baseline to 3 months, in $64 \%$ $v s$. $92 \%$ of patients (SRS alone vs. WBRT; $\mathrm{P}<0.001$ ). Even allowing for the costs associated with MRI surveillance and salvage therapy, SRS alone also demonstrates superior costeffectiveness over SRS+WBRT (40-42). Hence, SRS alone has become standard of care for fit patients with $\leq 4 \mathrm{BM}$, where close MRI surveillance is logistically possible (43-48).

In an attempt to avoid the neurocognitive decline associated with WBRT in the setting of resected BM (where historically, postoperative WBRT has been utilised), two randomized studies reporting in parallel have employed cavity SRS (49,50). The Mahajan et al. study [2017] (49) showed that, for patients who had 1-3 BM resected, postoperative SRS significantly lowered local recurrence, whereas the Brown et al. randomised trial [2017] (50) reported less cognitive decline in the SRS versus WBRT groups, with no difference in survival between the arms. These studies support the use of cavity SRS as a standard of care after resection of 1-3 BMs. 
Interestingly, the response of $\mathrm{BM}$ appears to be more favourable to SRS in breast cancer patients with HER2+ disease than in those with triple-negative breast cancer (TNBC) (Cagney et al., 2019).

Nguyen et al. [2019] (51) described an SRS technique (SPARE: spatially partitioned adaptive radiosurgery) for multiple BM that provides relatively lower off-target doses, including markedly less dose to the hippocampus. Such a single-isocenter SRS technique, delivered in $\leq 1 \mathrm{hr}$, may ultimately provide the best hippocampal sparing approach; SPARE is being prospectively tested in patients with 5-20 BM (NCT03775330).

\section{Systemic therapies: chemotherapy}

Traditional chemotherapy has been largely unsuccessful in the treatment of BM, and usually reserved until after the failure of local therapies. This has been due to a combination of factors, including the blood-brain barrier (BBB), blood-tumour barrier (BTB) and the presence of efficient CNS drug efflux pumps such as p-glycoprotein, which all combine to reduce local drug concentrations in $\mathrm{BM}$. Drug concentration heterogeneity in BM is also a factor (52). However, traditional chemotherapy responses do occur. For patients with BM from NSCLC, responses of the order of $10-15 \%$ in pretreated patients, to the following drug pairs have been recorded: cisplatin in combination with vinorelbine, pemetrexed or paclitaxel (53). Likewise, similar response rates are seen in breast cancer BM patients, but systemic chemotherapy is of little value in melanoma BM cases (53).

\section{Systemic therapies: targeted and immunotherapies, according to tumour type}

The major tumour types giving rise to BM, for which targeted and immunotherapies have made a significant contemporary impact, are (I) NSCLC, (II) melanoma and (III) breast cancer.

\section{NSCLC}

In NSCLC patients with EGFR mutations, first generation EGFR tyrosine kinase inhibitors (TKIs) such as gefitinib, icotinib and erlotinib showed activity in NSCLC patients with $\mathrm{BM}$ in retrospective studies and Phase II/III clinical trials (5). In-brain response rates were typically of the order of $50-80 \%$, generating overall survivals of 15-22 months $(54,55)$. Second-generation NSCLC-TKIs include neratinib, afatanib and dacomitinib. In two Phase III randomised trials of BM in patients with EGFR mutant NSCLC, using afatanib, an increase in PFS was observed in the study arm versus conventional chemotherapy arm, in all patients receiving afatanib. However, despite impressive response rates, response durations have been an issue with these drugs. Osimertinib, a third-generation EGFR-TKI, has improved CNS penetration and shows more durable response durations than earlier generation TKIs $(53,56)$ and can be considered the TKI of choice for EGFR-mutant lung cancer patients with BM. In the Phase III randomised FLAURA trial, osimertinib was compared with first line EGFR-TKIs as initial therapy. In the brain subgroup analysis, the CNS objective response rate was $91 \%$ in the osimertinib arm versus $68 \%$ for the first-line agents, while median PFS was not reached in the osimertinib arm (13.9 months for the first line TKIs) (57).

Other targeted therapies in NSCLC are directed against ALK rearrangement (ALKr) -positive NSCLC brain metastases. ALKr occurs in around $4-7 \%$ of NSCLC (58). A range of ALK-inhibitor TKIs are available, including the first generation inhibitor crizotinib, which, although it has limited BBB permeability, had higher in-brain activity that chemotherapy in a randomised Phase III trial (59), as did a later-generation ALK-TKI, ceritinib, which has greater BBB penetration (60). Lorlatinib is a third-generation ALK inhibitor TKI, which showed in a Phase II trial a $63 \%$ intracranial response rate after failure of at least one other ALK-TKI (61).

A variety of additional molecular targets are emerging for NSCLC, and some may prove to show efficacy for NSCLC $\mathrm{BM}$. These have been recently reviewed (5).

In recent years, immunotherapies have revolutionized the treatment of many cancer types. Immunotherapeutics have been raised against various cell surface antigens, including PD-1 (e.g., nivolumab and pembrolizumab), CTLA4 (e.g., ipilimumab), and PD-L1 (e.g., atezolizumab). Response rates are significantly lower for those on steroids [e.g., (46)], which is an impediment to ICI efficacy in BM. To maximise ICI efficacy, interest has increased in the use of the angiogenesis inhibitor bevacizumab as a steroidsparing approach to $\mathrm{BM}$-associated cerebral oedema, to maximise ICI responses. This is particularly pertinent in that ICI treatment can, in the case of an intracerebral response, be associated with peritumoural oedema (62), socalled pseudoprogression, which may be partially or wholly abrogated by an antiangiogenesis approach.

ICIs have shown emerging efficacy in patients with 
BM from subtypes of NSCLC not bearing druggable alterations. Pembrolizumab, the anti PD-1 monoclonal, was used in a Phase II trial of EGFR wild type NSCLC patients with BM. The intracranial response rate in those with $\mathrm{PD}$ L1 expression was $30 \%$, while none of those without PDL1 expression responded (63). Likewise, atezolizumab, an anti PD-L1 monoclonal, showed in a subgroup analysis of a Phase III randomised NSCLC trial, improved intracranial control compared to chemotherapy (64).

\section{Melanoma}

For melanoma patients with BM and BRAF mutations, targeted therapies have proved highly efficacious. BRAF status has been added to the melanoma-specific Graded Prognostic Assessment: BRAF mutation has a positive effect on prognosis, and melanoma BM patients in the best GPA group now have survivals ranging up to 36 months (65). BRAF mutations are the only molecular targets currently clinically druggable with TKIs in melanoma patients with BM. The BRAF inhibitors, dabrafenib and vemurafenib have in-brain response rates of $20-38 \%(66,67)$. Neither dabrafenib nor vemurafenib have been trialled head-tohead in melanoma patients with BM, but preclinical studies suggest that CNS penetration is superior for dabrafenib (68). Molecular combination approaches, based on their greater efficacy in systemic melanoma, have also been used in melanoma BM patients: in a seminal Phase II trial, COMBI$M B$, the mitogen-activated protein kinase (MAPK)inhibitor, trametinib, in conjunction with dabrafenib, yielded in-brain response rates as high as $55 \%(69)$. In patients in the most favourable cohort, intracranial control was $78 \%$. However, as for other tumour types, TKI response durations are often limited to less than 6 months [summarised in (53)]. Future targeted therapy trials should also incorporate QoL and NCF indices, especially in combination therapy trials.

ICIs have improved outcomes for melanoma patients with BM. In a Phase II study, ipilimumab, a CTLA4 inhibitor, achieved an $18 \%$ objective in-brain response rate in those melanoma BM patients not on steroids (70). Likewise, pembrolizumab, a PD-1 inhibitor, showed a $26 \%$ intra- and extra-cranial response rate, maintained at 24 months; most responders were PD-L1-high, whereas none of the non-responders were (71).

In melanoma BM patients, dual ICI therapy has achieved impressively high intracranial response rates. In the CHECKMATE 204 Phase II trial (12), melanoma BM patients could be pretreated with SRS or BRAF inhibitors, or were treatment-naïve (the majority); with a median follow-up of over 20 months, the intracranial response rate was $55 \%$ (26\% complete responses), with 70\% 2-year survivals, a massive improvement compared with preimmunotherapy times.

\section{Breast cancer}

In breast cancer BM patients, HER2+ cases have been the focus of most targeted therapy trials. The most established anti-HER2 therapy for systemic and newly diagnosed disease, trastuzumab, has minimal BBB penetration however (72). Despite this, some responses to anti-HER2+ monoclonal antibodies in HER2 + BM patients have been observed: high dose trastuzumab plus pertuzumab was delivered in the Phase II PATRICIA study (73). Ten percent of patients had an objective CNS response, while over 50\% achieved a clinical benefit (complete or partial response or stable disease for $>6$ months).

For HER2 + breast cancer cases with BM, the oral TKI lapatinib has been used mainly in conjunction with capecitabine, demonstrating in phase II trials response rates of $20-66 \%$ (depending on whether patients had had prior RT) (74). Other HER2-targeted TKIs include neratinib and tucatinib. HER2CLIMB was a randomised Phase III trial of tucatinib in combination with capecitabine and trastuzumab, versus capecitabine/trastuzumab doublet therapy in advanced HER2 + metastatic breast cancer (75). $\mathrm{BM}$ patients had longer progression free and overall survival in the tucatinib-containing regimen.

In the case of TNBC BM patients, ICIs have started to contribute to intracranial control. In the IMpassion130 Phase III Study, atezolizumab plus taxane was compared to taxane alone in metastatic TNBC patients, previously untreated and with PD-L1-positive cancers (76). Compared to the general study population, the hazard ratio for progression-free survival was similar for patients with BM. Characterisation of CNS responses are awaited from subsequent subgroup analyses.

ER/PR-positive, HER2-negative breast cancer has a lower predilection than other breast cancer subtypes to metastasize to the brain. However, BM are not uncommon in advanced disease. In this context, CDK4/6 inhibitors have been employed, where abemaciclib seems to have superior CNS penetration to palbociclib. In the JPBO Phase II Study, abemaciclib yielded a $6 \%$ intracranial overall response rate, but there was a $25 \%$ rate of clinical benefit (defined as above) (77). 


\section{Situations where targeted therapies may replace or delay $\mathbf{R T}$}

It has been suggested (78) that, based on recommendations from the National Comprehensive Cancer Network (NCCN), The European Society for Medical Oncology (ESMO) and the Japan Lung Cancer Society, cerebral irradiation can be avoided until disease progression occurs, in the case of asymptomatic NSCLC patients with EGFR mutations or ALK rearrangements (ALKr), as long as the appropriate TKIs and close MRI surveillance are available. Preferred TKIs are osimertinib for EGFR mutant patients, and alectinib, brigatinib or ceritinib for ALKr patients. Another situation where radiation may be omitted or delayed is in melanoma BM patients, with small, asymptomatic brain metastases, where ipilimumab/ nivolumab combination therapy could be considered as first-line therapy (12).

\section{Combination of targeted therapies and ICIs with SRS}

There are various theoretical rationales for drug-radiation combinations in the treatment of BM $(5,53,79-81)$. Such combinations could be considered with the intent of improving both local tumour control and distant intracranial disease control. Combinations may exert biological co-operation, with resultant treatment intensification. Furthermore, drug CNS penetration and hence efficacy may be enhanced by localised blood-brainand blood-metastasis-barrier disruption by irradiation, whilst p-glycoprotein degradation by irradiation may abrogate drug efflux from the irradiated area. For distant intracranial disease control, the different modalities can spatially co-operate; in the case of immunotherapies, abscopal effects can additionally occur, with radiation acting as an in situ personalised vaccine (82). De-intensification of SRS in lower risk disease by concomitant drug use might be considered, with reduction in SRS side-effects, e.g., radionecrosis, thereby improving the therapeutic ratio.

There have been many trials of radiotherapy and TKIs in NSCLC patients with BM [reviewed in (83)]. Most have employed WBRT, and have been Phase II or III trials; three meta-analyses are also available (with 980, 1,552 and 2,810 patients respectively) (84-86). All meta-analyses showed, in the radiation+TKI arms, greater response rates, CNS timeto-progression and overall survival. Although one reported no difference in serious adverse events between arms (84), the other two reported an increase in any adverse event in the radiation+TKI arms $(85,86)$. However, the latter studies showed that in general adverse events were tolerable. Hence, concurrent use of EGFR TKIs and radiation appears effective and tolerable. Remaining questions include the optimal sequencing of the two modalities and whether NSCLC TKIs increase the risk of radionecrosis after SRS radiotherapy. The same question remains for combination ICIs and SRS.

For ALK TKIs, there is currently minimal evidence for or against their combined use with irradiation; one retrospective study of ALKr NSCLC patients suggested an increase in ototoxicity with combined ALK TKIs and WBRT (87). For WBRT (where the globes receive radiation dose), care may need to be exerted because of potential overlapping ocular toxicity with crizotinib (83). Oto- and ocular-toxicity from the WBRT component might be circumvented by using VMAT.

Regarding drug-SRS combinations, it is informative to consider $12-$ month radionecrosis risk rates. For SRS alone, the risk is $5.3 \%$ (88). When all drugs combined are considered, the risk rises minimally, to $6.6 \%$, whereas it is $8.8 \%$ for all targeted therapies, as it is for PD-1/CTLA4 ICIs. For anti-HER2 monoclonals combined with SRS, the 12 -month radionecrosis risk is $9.0 \%$, peaking at $14 \%$ for EGFR-TKIs (88). Despite this, one meta-analysis showed no increased radionecrosis risk with EGFR-TKI combinations (84). A number of studies [including $(89,90)$ ] and including an international meta-analysis of individual patient data (79) showed clearly improved overall survival in BM patients when ICIs were administered concurrently with irradiation. Clearly, caution must be taken if ICIs are to be routinely and concurrently combined with SRS. Minimal prospective data on the risk of ICI-induced radionecrosis is available because the combination of SRS with ICIs is fairly new. Caution is especially pertinent in that radionecrosis is a delayed phenomenon, and future prospective studies will be required to better elucidate the efficacy and toxicity of concurrent and sequential ICI-SRS combinations.

\section{Summary and future}

Improved understanding of the biology of BM, including the brain- and BM-microenvironment (91,92), BBB and BTB (93), as well as the use of in vivo models (e.g., patientderived xenografts in immunodeficient mouse strains) (94) is expected to aid the development of novel diagnostic and therapeutic approaches to BM. Minimally invasive surgery 
will be increasingly used for obtaining tissue for molecular $\mathrm{BM}$ classification. Integration of such knowledge into prognostic models should improve patient selection for precision medicine approaches to BM patients, reduce the toxicity of therapy and hopefully extend the current largely poor prognosis for this disease.

In $\mathrm{BM}$ patients, new druggable targets are under investigation in breast cancer (e.g., CDK4/6 and ER signalling pathways, DNA repair pathways), in NSCLC (e.g., NTRK fusions, ROS1 rearrangements, KRAS and BRAF mutations) and in melanoma (e.g., MEK pathway) $(5,46,53)$. In vivo analysis of new candidate drugs will be facilitated by phase 0 clinical trials.

Liquid biopsies will almost certainly be increasingly used for $\mathrm{BM}$ diagnosis and monitoring of the response of $\mathrm{BM}$ to therapy. Such minimally invasive approaches will aid in optimising the personalised use of targeted- and immunotherapeutics. For example, for multiple tumour types, there is a discordance between the molecular phenotype of the peripheral and BM tumours in the same patient (53); liquid biopsies could avoid craniotomies in such cases. Advanced neuroimaging is expected to shed earlier light on therapy responses.

In the future, drugs, including immunotherapeutics and targeted therapies, with satisfactory CNS penetration and activity against BM, could increasingly and rationally be combined with focal BM therapies for the reasons mentioned previously. However, their incorporation into clinical use will require initial testing, in particular, characterisation of radionecrosis rates, in clinical trials. Improved integration and sequencing of focal BM therapies with targeted and immunotherapies should improve the therapeutic ratio.

\section{Acknowledgments}

The author thanks Dr. Richard Foster for thoughtful comments on the manuscript and acknowledges Associate Professor Mark Pinkham for helpful discussion on the drugradiation section of the manuscript.

Funding: None.

\section{Footnote}

Reporting Checklist: The author has completed the Narrative Review reporting checklist. Available at https://dx.doi. org/10.21037/atm-21-3665
Conflicts of Interest: The author has completed the ICMJE uniform disclosure form (available at https://dx.doi. org/10.21037/atm-21-3665). The author has no conflicts of interest to declare.

Ethical Statement: The author is accountable for all aspects of the work in ensuring that questions related to the accuracy or integrity of any part of the work are appropriately investigated and resolved.

Open Access Statement: This is an Open Access article distributed in accordance with the Creative Commons Attribution-NonCommercial-NoDerivs 4.0 International License (CC BY-NC-ND 4.0), which permits the noncommercial replication and distribution of the article with the strict proviso that no changes or edits are made and the original work is properly cited (including links to both the formal publication through the relevant DOI and the license). See: https://creativecommons.org/licenses/by-nc-nd/4.0/.

\section{References}

1. Available online: https://www.aihw.gov.au/reports/cancer/ cancer-data-in-australia/data

2. Gavrilovic IT, Posner JB. Brain metastases: epidemiology and pathophysiology. J Neurooncol 2005;75:5-14.

3. Barnholtz-Sloan JS, Sloan AE, Davis FG, et al. Incidence proportions of brain metastases in patients diagnosed (1973 to 2001) in the Metropolitan Detroit Cancer Surveillance System. J Clin Oncol 2004;22:2865-72.

4. Cagney DN, Martin AM, Catalano PJ, et al. Incidence and prognosis of patients with brain metastases at diagnosis of systemic malignancy: a population-based study. Neuro Oncol 2017;19:1511-21.

5. Soffietti R, Ahluwalia M, Lin N, et al. Management of brain metastases according to molecular subtypes. Nat Rev Neurol 2020;16:557-74.

6. Jakob JA, Bassett RL Jr, Ng CS, et al. NRAS mutation status is an independent prognostic factor in metastatic melanoma. Cancer 2012;118:4014-23.

7. Iuchi T, Shingyoji M, Itakura $M$, et al. Frequency of brain metastases in non-small-cell lung cancer, and their association with epidermal growth factor receptor mutations. Int J Clin Oncol 2015;20:674-9.

8. Eichler AF, Loeffler JS. Multidisciplinary management of brain metastases. Oncologist 2007;12:884-98.

9. Sperduto CM, Watanabe $\mathrm{Y}$, Mullan J, et al. A validation 
study of a new prognostic index for patients with brain metastases: the Graded Prognostic Assessment. J Neurosurg 2008;109 Suppl:87-9.

10. Karlsson B, Yamamoto M, Hanssens P, et al. Does Modern Management of Malignant Extracranial Disease Prolong Survival in Patients with $\geq 3$ Brain Metastases? World Neurosurg 2016;92:279-83.

11. Walker AE, Robins M, Weinfeld FD. Epidemiology of brain tumors: the national survey of intracranial neoplasms. Neurology 1985;35:219-26.

12. Tawbi HA, Forsyth PA, Algazi A, et al. Combined Nivolumab and Ipilimumab in Melanoma Metastatic to the Brain. N Engl J Med 2018;379:722-30.

13. Patchell RA, Tibbs PA, Regine WF, et al. Postoperative radiotherapy in the treatment of single metastases to the brain: a randomized trial. JAMA 1998;280:1485-9.

14. Arnold SM, Patchell RA. Diagnosis and management of brain metastases. Hematol Oncol Clin North Am 2001;15:1085-107, vii.

15. Kong X, Guan J, Yang Y, et al. A meta-analysis: Do prophylactic antiepileptic drugs in patients with brain tumors decrease the incidence of seizures? Clin Neurol Neurosurg 2015;134:98-103.

16. Soffietti R, Cornu P, Delattre JY, et al. EFNS Guidelines on diagnosis and treatment of brain metastases: report of an EFNS Task Force. Eur J Neurol 2006;13:674-81.

17. Chang SM, Messersmith H, Ahluwalia M, et al. Anticonvulsant Prophylaxis and Steroid Use in Adults With Metastatic Brain Tumors: ASCO and SNO Endorsement of the Congress of Neurological Surgeons Guidelines. J Clin Oncol 2019;37:1130-5.

18. Karnofsky DA, Burchenal JH. The clinical evaluation of chemotherapeutic agents in cancer. In: MacLeod CM, editor. Evaluation of chemotherapeutic agents. New York, NY: Columbia University Press, 1949:199-208.

19. Barker FG 2nd. Craniotomy for the resection of metastatic brain tumors in the U.S., 1988-2000: decreasing mortality and the effect of provider caseload. Cancer 2004;100:999-1007.

20. Patchell RA, Tibbs PA, Walsh JW, et al. A randomized trial of surgery in the treatment of single metastases to the brain. N Engl J Med 1990;322:494-500.

21. Vecht CJ, Haaxma-Reiche H, Noordijk EM, et al. Treatment of single brain metastasis: radiotherapy alone or combined with neurosurgery? Ann Neurol 1993;33:583-90.

22. Stark AM, Tscheslog H, Buhl R, et al. Surgical treatment for brain metastases: prognostic factors and survival in 177 patients. Neurosurg Rev 2005;28:115-9.

23. Brown PD, Ahluwalia MS, Khan OH, et al. Whole-
Brain Radiotherapy for Brain Metastases: Evolution or Revolution? J Clin Oncol 2018;36:483-91.

24. Kocher M, Soffietti R, Abacioglu U, et al. Adjuvant Whole-Brain Radiotherapy Versus Observation After Radiosurgery or Surgical Resection of One to Three Cerebral Metastases: Results of the EORTC 22952-26001 Study. J Clin Oncol 2011;29:134-41.

25. Mulvenna P, Nankivell M, Barton R, et al. Dexamethasone and supportive care with or without whole brain radiotherapy in treating patients with non-small cell lung cancer with brain metastases unsuitable for resection or stereotactic radiotherapy (QUARTZ): results from a phase 3, noninferiority, randomised trial. Lancet 2016;388:2004-14.

26. Hong AM, Fogarty GB, Dolven-Jacobsen K, et al. Adjuvant Whole-Brain Radiation Therapy Compared With Observation After Local Treatment of Melanoma Brain Metastases: A Multicenter, Randomized Phase III Trial. J Clin Oncol 2019;37:3132-41.

27. Brown PD, Jaeckle K, Ballman KV, et al. Effect of Radiosurgery Alone vs Radiosurgery With Whole Brain Radiation Therapy on Cognitive Function in Patients With 1 to 3 Brain Metastases: A Randomized Clinical Trial. JAMA 2016;316:401-9.

28. Chang EL, Wefel JS, Hess KR, et al. Neurocognition in patients with brain metastases treated with radiosurgery or radiosurgery plus whole-brain irradiation: a randomised controlled trial. Lancet Oncol 2009;10:1037-44.

29. Soffietti R, Kocher M, Abacioglu UM, et al. A European Organisation for Research and Treatment of Cancer phase III trial of adjuvant whole-brain radiotherapy versus observation in patients with one to three brain metastases from solid tumors after surgical resection or radiosurgery: quality-of-life results. J Clin Oncol 2013;31:65-72.

30. Monje ML, Mizumatsu S, Fike JR, et al. Irradiation induces neural precursor-cell dysfunction. Nat Med 2002;8:955-62.

31. Bálentová S, Hajtmanová E, Filova B, et al. Effect of Fractionated Irradiation on the Hippocampus in an Experimental Model. Klin Onkol 2015;28:191-9.

32. Dhermain F, Noël G, Antoni D, et al. Role of radiation therapy in brain metastases management. Cancer Radiother 2020;24:463-9.

33. Gondi V, Pugh SL, Tome WA, et al. Preservation of memory with conformal avoidance of the hippocampal neural stem-cell compartment during whole-brain radiotherapy for brain metastases (RTOG 0933): a phase II multi-institutional trial. J Clin Oncol 2014;32:3810-6.

34. Aizer AA, Lee EQ. Brain Metastases. Neurol Clin 
2018;36:557-77.

35. Redmond KJ, Hales RK, Anderson-Keightly H, et al. Prospective Study of Hippocampal-Sparing Prophylactic Cranial Irradiation in Limited-Stage Small Cell Lung Cancer. Int J Radiat Oncol Biol Phys 2017;98:603-11.

36. Brown PD, Gondi V, Pugh S, et al. Hippocampal Avoidance During Whole-Brain Radiotherapy Plus Memantine for Patients With Brain Metastases: Phase III Trial NRG Oncology CC001. J Clin Oncol 2020;38:1019-29.

37. Rapp SR, Case LD, Peiffer A, et al. Donepezil for Irradiated Brain Tumor Survivors: A Phase III Randomized Placebo-Controlled Clinical Trial. J Clin Oncol 2015;33:1653-9.

38. Attia A, Rapp SR, Case LD, et al. Phase II study of Ginkgo biloba in irradiated brain tumor patients: effect on cognitive function, quality of life, and mood. J Neurooncol 2012;109:357-63.

39. Aoyama H, Shirato H, Tago M, et al. Stereotactic radiosurgery plus whole-brain radiation therapy vs stereotactic radiosurgery alone for treatment of brain metastases: a randomized controlled trial. JAMA 2006;295:2483-91.

40. Lester-Coll NH, Dosoretz AP, Magnuson WJ, et al. Costeffectiveness of stereotactic radiosurgery versus wholebrain radiation therapy for up to 10 brain metastases. J Neurosurg 2016;125:18-25.

41. Lal LS, Byfield SD, Chang EL, et al. Cost-effectiveness analysis of a randomized study comparing radiosurgery with radiosurgery and whole brain radiation therapy in patients with 1 to 3 brain metastases. Am J Clin Oncol 2012;35:45-50.

42. Savitz ST, Chen RC, Sher DJ. Cost-effectiveness analysis of neurocognitive-sparing treatments for brain metastases. Cancer 2015;121:4231-9.

43. Hahn C, Kavanagh B, Bhatnagar A, et al. Choosing wisely: the American Society for Radiation Oncology's top 5 list. Pract Radiat Oncol 2014;4:349-55.

44. Sahgal A, Ruschin M, Ma L, et al. Stereotactic radiosurgery alone for multiple brain metastases? A review of clinical and technical issues. Neuro Oncol 2017;19:ii2-ii15.

45. Bertolini F, Spallanzani A, Fontana A, et al. Brain metastases: an overview. CNS Oncol 2015;4:37-46.

46. Suh JH, Kotecha R, Chao ST, et al. Current approaches to the management of brain metastases. Nat Rev Clin Oncol 2020;17:279-99.

47. Tosoni A, Ermani M, Brandes AA. The pathogenesis and treatment of brain metastases: a comprehensive review. Crit Rev Oncol Hematol 2004;52:199-215.
48. Rosenfelder N, Brada M. Integrated treatment of brain metastases. Curr Opin Oncol 2019;31:501-7.

49. Mahajan A, Ahmed S, McAleer MF, et al. Post-operative stereotactic radiosurgery versus observation for completely resected brain metastases: a single-centre, randomised, controlled, phase 3 trial. Lancet Oncol 2017;18:1040-8.

50. Brown PD, Ballman KV, Cerhan JH, et al. Postoperative stereotactic radiosurgery compared with whole brain radiotherapy for resected metastatic brain disease (NCCTG N107C/CEC.3): a multicentre, randomised, controlled, phase 3 trial. Lancet Oncol 2017;18:1049-60.

51. Nguyen TK, Sahgal A, Detsky J, et al. Single-Fraction Stereotactic Radiosurgery Versus Hippocampal-Avoidance Whole Brain Radiation Therapy for Patients With 10 to 30 Brain Metastases: A Dosimetric Analysis. Int J Radiat Oncol Biol Phys 2019;105:394-9.

52. Askoxylakis V, Arvanitis CD, Wong CSF, et al. Emerging strategies for delivering antiangiogenic therapies to primary and metastatic brain tumors. Adv Drug Deliv Rev 2017;119:159-74.

53. Achrol AS, Rennert RC, Anders C, et al. Brain metastases. Nat Rev Dis Primers 2019;5:5.

54. Ceresoli GL, Cappuzzo F, Gregorc V, et al. Gefitinib in patients with brain metastases from non-small-cell lung cancer: a prospective trial. Ann Oncol 2004;15:1042-7.

55. Welsh JW, Komaki R, Amini A, et al. Phase II trial of erlotinib plus concurrent whole-brain radiation therapy for patients with brain metastases from non-small-cell lung cancer. J Clin Oncol 2013;31:895-902.

56. Mok TS, Wu YL, Ahn MJ et al. Osimertinib or PlatinumPemetrexed in EGFR T790M-Positive Lung Cancer. N Engl J Med. 2017;376:629-40.

57. Reungwetwattana T, Nakagawa K, Cho BC, et al. CNS Response to Osimertinib Versus Standard Epidermal Growth Factor Receptor Tyrosine Kinase Inhibitors in Patients With Untreated EGFR-Mutated Advanced NonSmall-Cell Lung Cancer. J Clin Oncol 2018. [Epub ahead of print].

58. Chia PL, Mitchell P, Dobrovic A, et al. Prevalence and natural history of ALK positive non-small-cell lung cancer and the clinical impact of targeted therapy with ALK inhibitors. Clin Epidemiol 2014;6:423-32.

59. Shaw AT, Kim DW, Nakagawa K, et al. Crizotinib versus chemotherapy in advanced ALK-positive lung cancer. N Engl J Med 2013;368:2385-94.

60. Soria JC, Tan DSW, Chiari R, et al. First-line ceritinib versus platinum-based chemotherapy in advanced ALKrearranged non-small-cell lung cancer (ASCEND-4): 
a randomised, open-label, phase 3 study. Lancet 2017;389:917-29.

61. Solomon BJ, Besse B, Bauer TM, et al. Lorlatinib in patients with ALK-positive non-small-cell lung cancer: results from a global phase 2 study. Lancet Oncol 2018;19:1654-67.

62. Cohen JV, Alomari AK, Vortmeyer AO, et al. Melanoma Brain Metastasis Pseudoprogression after Pembrolizumab Treatment. Cancer Immunol Res 2016;4:179-82.

63. Goldberg SB, Schalper KA, Gettinger SN, et al. Pembrolizumab for management of patients with NSCLC and brain metastases: long-term results and biomarker analysis from a non-randomised, open-label, phase 2 trial. Lancet Oncol 2020;21:655-63.

64. Gadgeel SM, Lukas RV, Goldschmidt J, et al. Atezolizumab in patients with advanced non-small cell lung cancer and history of asymptomatic, treated brain metastases: Exploratory analyses of the phase III OAK study. Lung Cancer 2019;128:105-12.

65. Sperduto PW, Jiang W, Brown PD, et al. Estimating Survival in Melanoma Patients With Brain Metastases: An Update of the Graded Prognostic Assessment for Melanoma Using Molecular Markers (MelanomamolGPA). Int J Radiat Oncol Biol Phys 2017;99:812-6.

66. Long GV, Trefzer U, Davies MA, et al. Dabrafenib in patients with Val600Glu or Val600Lys BRAF-mutant melanoma metastatic to the brain (BREAK-MB): a multicentre, open-label, phase 2 trial. Lancet Oncol 2012;13:1087-95

67. McArthur GA, Maio M, Arance A, et al. Vemurafenib in metastatic melanoma patients with brain metastases: an open-label, single-arm, phase 2, multicentre study. Ann Oncol 2017;28:634-41.

68. Mittapalli RK, Vaidhyanathan S, Dudek AZ, et al. Mechanisms limiting distribution of the threonineprotein kinase B-RaF(V600E) inhibitor dabrafenib to the brain: implications for the treatment of melanoma brain metastases. J Pharmacol Exp Ther 2013;344:655-64.

69. Davies MA, Saiag P, Robert C, et al. Dabrafenib plus trametinib in patients with BRAFV600-mutant melanoma brain metastases (COMBI-MB): a multicentre, multicohort, open-label, phase 2 trial. Lancet Oncol 2017;18:863-73.

70. Margolin K, Ernstoff MS, Hamid O, et al. Ipilimumab in patients with melanoma and brain metastases: an openlabel, phase 2 trial. Lancet Oncol 2012;13:459-65.

71. Kluger HM, Chiang V, Mahajan A, et al. Long-Term Survival of Patients With Melanoma With Active Brain
Metastases Treated With Pembrolizumab on a Phase II Trial. J Clin Oncol 2019;37:52-60.

72. Lin NU, Amiri-Kordestani L, Palmieri D, et al. CNS metastases in breast cancer: old challenge, new frontiers. Clin Cancer Res 2013;19:6404-18.

73. Lin NU, Pegram M, Sahebjam S, et al. Pertuzumab Plus High-Dose Trastuzumab in Patients With Progressive Brain Metastases and HER2-Positive Metastatic Breast Cancer: Primary Analysis of a Phase II Study. J Clin Oncol 2021;39:2667-75.

74. Bachelot T, Romieu G, Campone M, et al. Lapatinib plus capecitabine in patients with previously untreated brain metastases from HER2-positive metastatic breast cancer (LANDSCAPE): a single-group phase 2 study. Lancet Oncol 2013;14:64-71.

75. Murthy RK, Loi S, Okines A, et al. Tucatinib, Trastuzumab, and Capecitabine for HER2-Positive Metastatic Breast Cancer. N Engl J Med 2020;382:597-609.

76. Schmid P, Rugo HS, Adams S, et al. Atezolizumab plus nab-paclitaxel as first-line treatment for unresectable, locally advanced or metastatic triple-negative breast cancer (IMpassion130): updated efficacy results from a randomised, double-blind, placebo-controlled, phase 3 trial. Lancet Oncol 2020;21:44-59.

77. Tolaney SM, Sahebjam S, Le Rhun E, et al. A Phase II Study of Abemaciclib in Patients with Brain Metastases Secondary to Hormone Receptor-Positive Breast Cancer. Clin Cancer Res 2020;26:5310-9.

78. Nishino M, Soejima K, Mitsudomi T. Brain metastases in oncogene-driven non-small cell lung cancer. Transl Lung Cancer Res 2019;8:S298-307.

79. Lehrer EJ, Peterson J, Brown PD, et al. Treatment of brain metastases with stereotactic radiosurgery and immune checkpoint inhibitors: An international meta-analysis of individual patient data. Radiother Oncol 2019;130:104-12.

80. Ahluwalia MS, Winkler F. Targeted and immunotherapeutic approaches in brain metastases. Am Soc Clin Oncol Educ Book 2015;67-74.

81. Franchino F, Rudà R, Soffietti R. Mechanisms and Therapy for Cancer Metastasis to the Brain. Front Oncol 2018;8:161.

82. Golden EB, Marciscano AE, Formenti SC. Radiation Therapy and the In Situ Vaccination Approach. Int J Radiat Oncol Biol Phys 2020;108:891-8.

83. Economopoulou P, Mountzios G. Non-small cell lung cancer (NSCLC) and central nervous system (CNS) metastases: role of tyrosine kinase inhibitors (TKIs) and evidence in favor or against their use with concurrent cranial radiotherapy. Transl 
Lung Cancer Res 2016;5:588-98.

84. Luo S, Chen L, Chen X, et al. Evaluation on efficacy and safety of tyrosine kinase inhibitors plus radiotherapy in NSCLC patients with brain metastases. Oncotarget 2015;6:16725-34.

85. Jiang T, Min W, Li Y, et al. Radiotherapy plus EGFR TKIs in non-small cell lung cancer patients with brain metastases: an update meta-analysis. Cancer Med 2016;5:1055-65.

86. Wang X, Xu Y, Tang W, et al. Efficacy and Safety of Radiotherapy Plus EGFR-TKIs in NSCLC Patients with Brain Metastases: A Meta-Analysis of Published Data. Transl Oncol 2018;11:1119-27.

87. Nakashima T, Nonoshita T, Hirata H, et al. Adverse Events of Concurrent Radiotherapy and ALK Inhibitors for Brain Metastases of ALK-Rearranged Lung Adenocarcinoma. In Vivo 2020;34:247-53.

88. Kim JM, Miller JA, Kotecha R, et al. The risk of radiation necrosis following stereotactic radiosurgery with concurrent systemic therapies. J Neurooncol 2017;133:357-68.

Cite this article as: McKay MJ. Brain metastases: increasingly precision medicine-a narrative review. Ann Transl Med 2021;9(21):1629. doi: 10.21037/atm-21-3665
89. Chen L, Douglass J, Kleinberg L, et al. Concurrent Immune Checkpoint Inhibitors and Stereotactic Radiosurgery for Brain Metastases in Non-Small Cell Lung Cancer, Melanoma, and Renal Cell Carcinoma. Int J Radiat Oncol Biol Phys 2018;100:916-25.

90. Pike LRG, Bang A, Ott P, et al. Radiation and PD-1 inhibition: Favorable outcomes after brain-directed radiation. Radiother Oncol 2017;124:98-103.

91. Steeg PS, Camphausen KA, Smith QR. Brain metastases as preventive and therapeutic targets. Nat Rev Cancer 2011;11:352-63.

92. Lah TT, Novak M, Breznik B. Brain malignancies: Glioblastoma and brain metastases. Semin Cancer Biol 2020;60:262-73.

93. Arvanitis CD, Ferraro GB, Jain RK. The blood-brain barrier and blood-tumour barrier in brain tumours and metastases. Nat Rev Cancer 2020;20:26-41.

94. Lowery FJ, Yu D. Brain metastasis: Unique challenges and open opportunities. Biochim Biophys Acta Rev Cancer 2017;1867:49-57. 